\title{
SCNS. 09. Pre-clinical evidence of antimaniac action by angiotensin II receptor blocker.
}

GOMES, J.A.S ${ }^{1}$, TOMAZ, V.S², SOUZA, G.C ${ }^{1}$, CAVALCANTE, L.M¹, MACÊDO, D. ${ }^{1}$

1Departamento de Fisiologia e Farmacologia, Faculdade de Medicina, Universidade Federal do Ceará.

2Departamento de Microbiologia Médica, Faculdade de Medicina, Universidade Federal do Ceará.

Introduction: The Bipolar Disorder (BD), a chronic psychiatric disorder, is highly prevalent and associated with functional disability and with excessive costs to the health system. The renin-angiotensin system (RAS) has been studied concerning neurological diseases, and the role of angiotensin II is being currently recognized as an important factor in anxiety and mood disorders. The deregulation of SRA brain is associated with the formation of reactive oxygen species, activation of pro-inflammatory pathways, reduced neuroplasticity and mitochondrial dysfunction. It is noteworthy that all these events are related to the pathophysiology of BD. Objectives: To evaluate the effect of candesartan (CDS) in an animal model of mania induced by d-amphetamine (AMPH). Methods: Adult male Swiss mice (20-25 g, $\mathrm{n}=6-8$ per group) were submitted to two treatment protocols. In the prevention protocol, animals received CDS $(0.1 ; 0.3 ; 1$ or $3 \mathrm{mg} / \mathrm{kg} /$ day), lithium (47.5 $\mathrm{mg} / \mathrm{kg} / \mathrm{day}$ ) or vehicle for 14 days and between the 8th and 14th day mice received AMPH ( $2 \mathrm{mg} / \mathrm{kg} /$ day ip) or saline. In the reversal protocol, AMPH or saline was administered for 14 days and between the 8th and 14th day the animals were treated with CDS, lithium or vehicle. The effect of CDS was evaluated on 14th day by examining the exploratory behavior in the open field, a test that is used for pre-clinical study of anti-manic drugs. Neurochemical tests were conducted to evaluate the oxidative stress, assessing reduced glutathione (GSH) in the prefrontal cortex (PF), hippocampus (HPC) and striatum (ST). The statistical analysis was performed by ANOVA followed by Student's-Newman-Keuls's test, considering $\mathrm{p}<0.05$ as significant. Results: In both treatment protocols, there was an increase in locomotor activity in the groups that received only AMPH, which was prevented and reversed by CDS, whose results were similar to the control group and the animals that received AMPH + lithium. AMPH reduced GSH in HPC and PF in prevention and in HPC, PF and ST. GSH brain levels in CDS-treated animals increased in HPC in the prevention and in PF, HPC and ST in the reversal protocol. Conclusions: CDS, similarly to $\mathrm{Li}$, is effective in reversing and preventing $\mathrm{AMPH}$-induced behavioral and neurochemical alterations. The results suggest an anti-maniac action of CDS and propose a novel, safe and highly promising therapeutic approach for the BD.

Keywords: Bipolar Disorder, Mania, Amphetamine, Candesartan

GOMES, J.A.S.; TOMAZ, V.S.; SOUZA, G.C.; CAVALCANTE, L.M.; MACÊDO, D. 2013. Pre-clinical evidence of antimaniac action by angiotensin II receptor blocker, p.19. In: Oriá, Reinaldo Barreto; Andrade, Geanne Matos de; Bruin, Veralice Meireles S. de. I International Symposium in Neuroscience Meeting [Blucher Neuroscience Proceedings n.1 v.1]. São Paulo: Blucher, 2014, http://dx.doi.org/10.5151/isnm-sine14 